\title{
DIVERGENCE TERMS IN THE SUPERTRACE HEAT ASYMPTOTICS FOR THE DE RHAM COMPLEX ON A MANIFOLD WITH BOUNDARY
}

\author{
P. GILKEY ${ }^{1,2, \alpha, \beta}$, K. KIRSTEN ${ }^{2, \beta, \gamma}$, AND D. VASSILEVICH ${ }^{2, \beta}$
}

\begin{abstract}
We use invariance theory to determine the coefficient $a_{m+1, m}^{d+\delta}$ in the super trace for the twisted de Rham complex with absolute boundary conditions.
\end{abstract}

\section{INTRODUCTION}

Let $(M, g)$ be a compact Riemannian manifold of dimension $m$ with smooth, non-empty boundary $\partial M$. Let $\phi \in C^{\infty}(M)$ be an auxiliary smooth function called the dilaton. Let $d_{\phi}:=e^{-\phi} d e^{\phi}$ and let $\delta_{\phi, g}:=e^{\phi} \delta_{g} e^{-\phi}$ be the twisted exterior derivative and the co-derivative, respectively, on the space of smooth differential forms. The twisted or Witten Laplacian is given by:

$$
\Delta_{\phi, g}^{p}:=d_{\phi} \delta_{\phi, g}+\delta_{\phi, g} d_{\phi} \quad \text { on } \quad C^{\infty}\left(\Lambda^{p}(M)\right) .
$$

This operator appears in the study of quantum $p$ form fields interacting with a background dilaton [13, 21]. It has also been used in supersymmetric quantum mechanics 11] and in Morse theory [23].

We impose absolute boundary conditions $\mathcal{B}_{a}$, see [12] for details. Let $\Delta_{\phi, g, \mathcal{B}_{a}}^{p}$ be the associated realization. We need not consider relative boundary conditions $\mathcal{B}_{r}$ as the Hodge $\star$ operator intertwines $\Delta_{\phi, g, \mathcal{B}_{a}}^{p}$ and $\Delta_{-\phi, g, \mathcal{B}_{r}}^{m-p}$ if $M$ is orientable [13. These boundary conditions are motivated by the Hodge-de Rham theorem which shows

$$
\operatorname{ker}\left(\Delta_{\phi, g, \mathcal{B}_{a}}^{p}\right)=H^{p}(M) .
$$

The fundamental solution $e^{-t \Delta_{\phi, g, \mathcal{B}_{a}}^{p}}$ of the heat equation is an infinitely smoothing operator which is of trace class. Let $f \in C^{\infty}(M)$ be a smooth smearing function. Work of Greiner and Seeley [14, 19] shows there is a complete asymptotic expansion

$$
\operatorname{Tr}_{L^{2}}\left(f e^{-t \Delta_{\phi, g, \mathcal{B}_{a}}^{p}}\right) \sim \sum_{n \geq 0} a_{n, m}\left(f, \Delta_{\phi, g}^{p}, \mathcal{B}_{a}\right) t^{(n-m) / 2} \quad \text { as } \quad t \downarrow 0 .
$$

The heat trace invariants $a_{n, m}(\cdot)$ are locally computable. Let $\nabla_{e_{m}}^{k} f$ be the $k^{\text {th }}$ covariant derivative of $f$ with respect to the inward unit normal $e_{m}$ on $\partial M$. Let $d x$

Key words and phrases. Heat trace asymptotics, twisted de Rham complex, Witten Laplacian, invariants of the orthogonal group.

2000 Mathematics Subject Classification: 58J50.

${ }^{1}$ Research partially supported by the Mittag-Leffler Institute (Stockholm, Sweden).

${ }^{2}$ Research partially supported by the MPI (Leipzig, Germany).

${ }^{\alpha}$ Mathematics Department, University of Oregon, Eugene Or 97403 USA.

${ }^{\beta}$ Max-Planck-Institute for Mathematics in the Sciences, Inselstrasse 22, 04103 Leipzig Germany.

$\gamma$ Department of Mathematics, Baylor University, Waco, TX 76798 USA. 
and $d y$ be the Riemannian measures on $M$ and on $\partial M$, respectively. There exist local invariants $a_{n, m}\left(x, \Delta_{\phi, g}^{p}\right)$ and $a_{n, m, k}\left(y, \Delta_{\phi, g}^{p}, \mathcal{B}_{a}\right)$ so that

$$
\begin{aligned}
a_{n, m}\left(f, \Delta_{\phi, g}^{p}, \mathcal{B}_{a}\right) & =\int_{M} f(x) a_{n, m}\left(x, \Delta_{\phi, g}^{p}\right) d x \\
& +\sum_{k} \int_{\partial M} \nabla_{e_{m}}^{k} f(y) \cdot a_{n, m, k}\left(y, \Delta_{\phi, g}^{p}, \mathcal{B}_{a}\right) d y .
\end{aligned}
$$

The interior invariants vanish if $n$ is odd; the boundary invariants are generically non-zero for all $n \geq 1$. The presence of the smearing function $f$ localizes the problem and permits the recovery of divergence terms which would otherwise be lost. The presence of terms involving $\nabla_{e_{m}}^{k} f$ shows the kernel function for the fundamental solution of the heat equation behaves asymptotically like a distribution near the boundary as $t \downarrow 0$. Define the local supertrace heat asymptotics by setting:

$$
\begin{aligned}
& a_{n, m}^{d+\delta}(\phi, g)(x):=\sum_{p}(-1)^{p} a_{n, m}\left(x, \Delta_{\phi, g}^{p}\right), \\
& a_{n, m, k}^{d+\delta}(\phi, g)(y):=\sum_{p}(-1)^{p} a_{n, m, k}\left(y, \Delta_{\phi, g}^{p}, \mathcal{B}_{a}\right) .
\end{aligned}
$$

Let $\chi(M)$ be the Euler-Poincaré characteristic of $M$. If $f=1$ and if $\phi$ satisfies Neumann boundary conditions, then 13

$$
\sum_{p}(-1)^{p} \operatorname{Tr}_{L^{2}}\left(e^{-t \Delta_{\phi, g, \mathcal{B}_{a}}^{p}}\right)=\chi(M) .
$$

Equating terms in the asymptotic series yields:

$$
\int_{M} a_{n, m}^{d+\delta}(\phi, g)(x) d x+\int_{\partial M} a_{n, m, 0}^{d+\delta}(\phi, g)(y) d y=\left\{\begin{array}{ll}
\chi(M) & \text { if } n=m, \\
0 & \text { if } n \neq m
\end{array} .\right.
$$

The local index density has been computed in this setting [13]. Let indices $i, j, \ldots$ range from 1 to $m$ and index a local orthonormal frame for the tangent bundle of $M$. Let $R_{i j k l}$ be the associated components of the Riemann curvature tensor with the sign convention that $R_{1221}=+1$ on the unit sphere $S^{2} \subset \mathbb{R}^{3}$. Near the boundary, normalize the choice of the orthonormal frame so $e_{m}$ is the inward unit geodesic normal. Let indices $a, b, \ldots$ range from 1 to $m-1$ and index the induced orthonormal frame for the tangent bundle of the boundary. Let $L_{a b}$ be the components of the second fundamental form.

We adopt the Einstein convention and sum over repeated indices. Let

$$
\varepsilon_{U}^{V}:=g\left(e_{u_{1}} \wedge \ldots \wedge e_{u_{\mu}}, e_{v_{1}} \wedge \ldots \wedge e_{v_{\mu}}\right)
$$

be the totally anti-symmetric tensor. Let $I$ and $J$ be $m$ tuples of indices indexing an orthonormal frame for $T(M)$ and let $A$ and $B$ be $m-1$ tuples of indices indexing an orthonormal frame for $T(\partial M)$. Set

$$
\begin{aligned}
& \mathcal{R}_{J, s}^{I, t}:=R_{i_{s} i_{s+1} j_{s+1} j_{s}} \ldots R_{i_{t-1} i_{t} j_{t} j_{t-1}}, \\
& \mathcal{R}_{B, s}^{A, t}:=R_{a_{s} a_{s+1} b_{s+1} b_{s}} \ldots R_{a_{t-1} a_{t} b_{t} b_{t-1}}, \\
& \mathcal{L}_{B, t}^{A, t}:=L_{a_{s} b_{s}} \ldots L_{a_{t} b_{t}} .
\end{aligned}
$$

Since the empty product is 1 , we set $\mathcal{R}_{J, s}^{I, t}=1, \mathcal{R}_{B, s}^{A, t}=1$, and $\mathcal{L}_{B, s}^{A, t}=1$ if $t<s$.

We refer to 13 for the proof of the following result. It establishes vanishing theorems which generalize previous results of [2, 10, 11, 18, to the twisted setting. It also identifies the local index density in the twisted setting.

Theorem 1.1. (1) If $n$ is odd or if $n<m$, then $a_{n, m}^{d+\delta}(\phi, g)=0$.

(2) If $m$ is odd, then $a_{n, m}^{d+\delta}(0, g)=0$ for any $n$.

(3) If $n-k<m$, then $a_{n, m, k}^{d+\delta}(\phi, g)=0$. 
(4) $a_{2 \bar{m}, 2 \bar{m}}^{d+\delta}(\phi, g)=\frac{1}{\pi^{\bar{m}} 8^{\bar{m}} \bar{m} !} \varepsilon_{J}^{I} \mathcal{R}_{J, 1}^{I, m}$.

(5) $a_{m, m, 0}^{d+\delta}(\phi, g)=\sum_{k} \frac{1}{\pi^{k} 8^{k} k !(m-1-2 k) ! \operatorname{vol}\left(S^{m-1-2 k}\right)} \varepsilon_{B}^{A} \mathcal{R}_{B, 1}^{A, 2 k} \mathcal{L}_{B, 2 k+1}^{A, m-1}$.

The fact that the local index density is not dependent on the dilaton field has important physical consequences [13. One can also combine Equation (1.a) with Theorem 1.1 to obtain a heat equation proof of the Chern-Gauss-Bonnet theorem 8. 9] for manifolds with boundary:

$$
\begin{aligned}
\chi\left(M^{2 \bar{m}}\right) & =\int_{M} \frac{1}{\pi^{m} 8^{m} \bar{m} !} \varepsilon_{J}^{I} \mathcal{R}_{J, 1}^{I, m} d x \\
& +\sum_{k} \int_{\partial M} \frac{1}{\pi^{k} 8^{k} k !(2 \bar{m}-1-2 k) ! \operatorname{vol}\left(S^{2 \bar{m}-1-2 k}\right)} \varepsilon_{B}^{A} \mathcal{R}_{B, 1}^{A, 2 k} \mathcal{L}_{B, 2 k+1}^{A, 2 \bar{m}-1} d y, \\
\chi\left(M^{2 \bar{m}+1}\right) & =\sum_{k} \int_{\partial M} \frac{1}{\pi^{k} 8^{k} k !(2 \bar{m}-2 k) ! \operatorname{vol}\left(S^{2 \bar{m}-2 k}\right)} \varepsilon_{B}^{A} \mathcal{R}_{B, 1}^{A, 2 k} \mathcal{L}_{B, 2 k+1}^{A, 2 \bar{m}} d y .
\end{aligned}
$$

By Theorem 1.1] the first non-trivial 'divergence' terms can first arise in the supertrace when $n=m+1$. Let ';' and ':' denote multiple covariant differentiation with respect to the Levi-Civita connections on $M$ and on $\partial M$, respectively. By Theorem 1.1] $a_{m+1, m}^{d+\delta}(\phi, g)=0$ if $m$ is even. Furthermore $a_{m+1, m, k}^{d+\delta}(\phi, g)=0$ if $k \geq 2$. The following is the main result of this paper:

Theorem 1.2. (1) $a_{2 \bar{m}+2,2 \bar{m}+1}^{d+\delta}(\phi, g)=\frac{1}{\sqrt{\pi} \pi^{\bar{m}} 8^{\bar{m}} \bar{m} !} \varepsilon_{J}^{I} \phi_{; i_{1} j_{1}} \mathcal{R}_{J, 2}^{I, m}$.

(2) $a_{m+1, m, 0}^{d+\delta}(\phi, g)=\sum_{k} \frac{1}{\sqrt{\pi} \pi^{k} 8^{k} k ! \operatorname{vol}\left(S^{m-2 k-2}\right)(m-2 k-2) !} \varepsilon_{B}^{A} \phi_{; a_{1} b_{1}} \mathcal{R}_{B, 2}^{A, 2 k+1} \mathcal{L}_{B, 2 k+2}^{A, m-1}$ $+\sum_{2 k<m-3} \frac{1}{2 \sqrt{\pi} \pi^{k} 8^{k} k ! \operatorname{vol}\left(S^{m-2 k-2}\right)(m-2 k-2) !}$

$$
\text { - } \varepsilon_{B}^{A}\left\{\mathcal{R}_{B, 1}^{A, 2 k} R_{a_{2 k+1} a_{2 k+2} b_{2 k+2} m} \mathcal{L}_{B, 2 k+3}^{A, m-1}\right\}: b_{2 k+1},
$$

(3) $a_{m+1, m, 1}^{d+\delta}(\phi, g)=\sum_{k} \frac{\sqrt{\pi}}{8^{k} \pi^{k} k ! \operatorname{vol}\left(S^{m-2 k}\right)(m-2 k) !} \varepsilon_{B}^{A} \mathcal{R}_{B, 1}^{A, 2 k} \mathcal{L}_{B, 2 k+1}^{A, m-1}$.

Let $M$ be a closed manifold. The local index density for the untwisted de Rham complex was identified in dimension 2 by McKean and Singer [16] and in arbitrary dimensions by Atiyah, Bott, and Patodi [2], by Gilkey [10, and by Patodi [18. The case of manifolds with boundary was studied in [11. We also refer to 3, 4, 17] for other treatments of the local index theorem.

Patodi's approach involved a direct calculation analyzing cancellation formulas for the fundamental solution of the heat equation. Atiyah, Bott, and Patodi used invariance theory to identify the local index density for the twisted signature and twisted spin complexes. They then expressed the de Rham complex locally in terms of the spin complex twisted by a suitable coefficient bundle. Neither of these approaches seems particularly well adapted to the twisted setting. In particular, since the operator $d_{\phi}$ relies on the $\mathbb{Z}$ grading of the de Rham complex, it is not described in terms of an operator on the twisted signature or spin complexes. Thus we choose in [13] to generalize the approach of [10] to determine the local index density for the twisted de Rham complex.

There are explicit combinatorial formulas 6, 7. 15 for the heat trace invariants of order $n \leq 5$, see the discussion in Section 2 for further details. However, these formulas become very complicated and it seems hopeless to prove Theorem 1.2 by an explicit computation.

The approach taken by Gilkey in [10] suffered from the disadvantage that the techniques involved were rather ad hoc and cumbersome as they did not make full use of the machinery of invariance theory developed by H. Weyl 22. In the present paper, we use both the first and second main theorems of invariance theory; this is 
the crucial new feature of our analysis. Let

$$
\begin{array}{ll}
\mathcal{E}_{m+1, m} & :=\varepsilon_{I}^{J} \phi_{; i_{1} j_{1}} \mathcal{R}_{J, 2}^{I, m}, \\
\mathcal{F}_{m-1, m}^{k} & :=\varepsilon_{B}^{A} \mathcal{R}_{B, 1}^{A, 2 k} \mathcal{L}_{B, 2 k+1}^{A, m-1} \\
\mathcal{F}_{m, m}^{1, k} & :=\varepsilon_{B}^{A} \mathcal{R}_{B, 1}^{A, 2 k} \phi_{; a_{2 k+1} b_{2 k+1}} \mathcal{L}_{B, 2 k+2}^{A, m-1} \\
\mathcal{F}_{m, m}^{2, k} & :=\varepsilon_{B}^{A} \mathcal{R}_{B, 1}^{A, 2 k} \phi_{; a_{2 k+1}} \phi_{; b_{2 k+1}} \mathcal{L}_{B, 2 k+2}^{A, m-1}, \\
\mathcal{F}_{m, m}^{3, k} & :=\varepsilon_{B}^{A}\left\{\mathcal{R}_{B, 1}^{A, 2 k} R_{a_{2 k+1} a_{2 k+2} b_{2 k+2} m} \mathcal{L}_{B, 2 k+3}^{A, m-1}\right\}_{: b_{2 k+1}}
\end{array}
$$

Lemma 1.3. There exist universal constants so that:

(1) If $m$ is odd, then $a_{m+1, m}^{d+\delta}(\phi, g)=c_{m+1, m} \mathcal{E}_{m+1, m}$.

(2) $a_{m+1, m, 1}^{d+\delta}(\phi, g)=\sum_{k} c_{m+1, m, 1}^{k} \mathcal{F}_{m-1, m}^{k}$.

(3) $a_{m+1, m, 0}^{d+\delta}(\phi, g)=\sum_{i, k} c_{m+1, m, 0}^{i, k} \mathcal{F}_{m, m}^{i, k}$.

This reduces the proof of Theorem 1.2 to the evaluation of the unknown universal coefficients. Here is a brief guide to the remainder of the paper. In Section 2 we review the properties of the heat trace invariants which we will need. In Section 3 . we use invariance theory to establish Lemma 1.3 In Section 4 we employ product formulas, special case calculations, and functorial properties to derive some technical results concerning the universal coefficients of Lemma 1.3 We then combine these results to complete the proof of Theorem 1.2 in Section $[5$

\section{Formulas for the heAt trace asymptotics}

Let $D$ be an arbitrary operator of Laplace type on a vector bundle $V$. There is a canonical connection 12] $\nabla$ on $V$ which we use to differentiate tensors of all types and a canonical endomorphism $E$ of $V$ so that

$$
D u=-\left(u_{; i i}+E u\right) \text {. }
$$

We impose mixed boundary conditions. Let $\chi$ be an endomorphism of $\left.V\right|_{\partial M}$ so $\chi^{2}=1$. Decompose $\chi=\Pi_{+}-\Pi_{-}$where $\Pi_{ \pm}:=\frac{1}{2}(\operatorname{Id} \pm \chi)$ are the projections on the \pm 1 eigenspaces of $\chi$. Let $S$ be an auxiliary endomorphism of $\Pi_{+}$. We extend $\chi$ and $S$ to be parallel with respect to the geodesic normal vector field $e_{m}$ near $\partial M$. We impose Robin boundary conditions on $V_{+}:=\operatorname{Range}\left(\Pi_{+}\right)$and Dirichlet boundary conditions on $V_{-}:=$Range $\left(\Pi_{-}\right)$to define the mixed boundary operator:

$$
\mathcal{B}:=\left.\left\{\Pi_{+}\left(\nabla_{e_{m}}+S\right) \oplus \Pi_{-}\right\}\right|_{\partial M} .
$$

Let $\Omega_{i j}$ be the components of the curvature endomorphism defined by $\nabla$. We refer to [6] for the proof of the following result which expresses the heat trace asymptotics in terms of this formalism for $n \leq 3$ :

Lemma 2.1. (1) $a_{0}(f, D, \mathcal{B})=(4 \pi)^{-m / 2} \int_{M} \operatorname{Tr}(f \mathrm{Id}) d x$.

(2) $a_{1}(f, D, \mathcal{B})=(4 \pi)^{-(m-1) / 2} \frac{1}{4} \int_{\partial M} \operatorname{Tr}(f \chi) d y$.

(3) $a_{2}(f, D, \mathcal{B})=(4 \pi)^{-m / 2} \frac{1}{6} \int_{M} \operatorname{Tr}\left\{f\left(6 E+R_{i j j i} \mathrm{Id}\right)\right\} d x$ $+(4 \pi)^{-m / 2} \frac{1}{6} \int_{\partial M} \operatorname{Tr}\left\{f\left(2 L_{a a} \mathrm{Id}+12 S\right)+3 f_{; m} \chi\right\} d y$.

(4) $a_{3}(f, D, \mathcal{B})=(4 \pi)^{-(m-1) / 2} \frac{1}{384} \int_{\partial M} \operatorname{Tr}\left\{f\left(96 \chi E+16 \chi R_{i j j i}+8 \chi R_{a m a m}\right.\right.$ $+\left[13 \Pi_{+}-7 \Pi_{-}\right] L_{a a} L_{b b}+\left[2 \Pi_{+}+10 \Pi_{-}\right] L_{a b} L_{a b}+96 S L_{a a}+192 S^{2}$

$\left.\left.-12 \chi_{: a} \chi_{: a}\right)+f_{; m}\left(\left[6 \Pi_{+}+30 \Pi_{-}\right] L_{a a}+96 S\right)+24 \chi f_{; m m}\right\} d y$. 
Similar formulas are available $[6,7,15,20$, for $n=4,5$. What is crucial to our analysis, however, is the general form of these expressions. They are the trace of certain non-commutative polynomials in the covariant derivatives of the variables $\{R, E, \Omega, S, L, \chi\}$ with indices contracted in pairs.

To apply Lemma 2.1 to the setting at hand, we must identify the structures which are involved for the twisted Laplacian. Let $\mathfrak{e}_{i}: \omega \rightarrow e_{i} \wedge \omega$ be left exterior multiplication by the covector $e_{i}$ and let $\mathfrak{i}_{i}$ be the dual operator, left interior multiplication by $e_{i}$. Let $\gamma_{i}=\mathfrak{e}_{i}-\mathfrak{i}_{i}$ give the associated Clifford module structure on the exterior algebra. Extend the Levi-Civita connection to act on tensors of all types and let $\Omega_{i j}$ be the associated curvature operator.

Lemma 2.2. (1) $\Delta_{\phi, g}=\Delta_{g}+\phi_{; i} \phi_{; i} \cdot \operatorname{Id}+\phi_{; j i}\left(\mathfrak{e}_{i} \mathfrak{i}_{j}-\mathfrak{i}_{j} \mathfrak{e}_{i}\right)$.

(2) The Levi-Civita connection is the connection associated to $\Delta_{\phi, g}$.

(3) $E_{\phi, g}:=-\frac{1}{2} \gamma_{i} \gamma_{j} \Omega_{i j}-\phi_{; i} \phi_{; i}-\phi_{; j i}\left(\mathfrak{e}_{i} \mathfrak{i}_{j}-\mathfrak{i}_{j} \mathfrak{e}_{i}\right)$ is the endomorphism for $\Delta_{\phi, g}$.

(4) Absolute boundary conditions are defined by taking

$$
\chi:=\left\{\begin{array}{ll}
+1 & \text { on } \Lambda(\partial M) \\
-1 & \text { on } \Lambda(\partial M)^{\perp}
\end{array}\right\} \quad \text { and } S:=\left\{\begin{aligned}
-L_{a b} \mathfrak{e}_{b} \mathfrak{i}_{a} & \text { on } \Lambda(\partial M) \\
0 & \text { on } \Lambda(\partial M)^{\perp}
\end{aligned}\right\} .
$$

(5) $\chi_{: a}=2 L_{a b}\left(\mathfrak{e}_{b} \mathfrak{i}_{m}+\mathfrak{e}_{m} \mathfrak{i}_{b}\right)$.

Proof. The classical formula $d+\delta_{g}=\mathfrak{e}_{i} \nabla_{e_{i}}-\mathfrak{i}_{j} \nabla_{e_{j}}$ extends to the twisted setting:

$$
d_{\phi}+\delta_{\phi, g}=\mathfrak{e}_{i} \nabla_{e_{i}}+\mathfrak{e}_{i} \phi_{; i}-\mathfrak{i}_{i} \nabla_{e_{i}}+\mathfrak{i}_{i} \phi_{; i} .
$$

We use the commutation rules $\mathfrak{e}_{i} \mathfrak{i}_{j}+\mathfrak{i}_{j} \mathfrak{e}_{i}=\delta_{i j}$, the fact that $\nabla \mathfrak{e}=0$, and the fact that $\nabla \mathfrak{i}=0$ to prove Assertion (1) by computing:

$$
\begin{aligned}
\Delta_{\phi, g}= & \Delta_{g}+\mathfrak{e}_{i} \nabla_{e_{i}} \mathfrak{i}_{j} \phi_{; j}+\mathfrak{i}_{j} \phi_{; j} \mathfrak{e}_{i} \nabla_{e_{i}}-\mathfrak{i}_{i} \nabla_{e_{i}} \mathfrak{e}_{j} \phi_{; j} \\
& -\mathfrak{e}_{j} \phi_{; j} \mathfrak{i}_{i} \nabla_{e_{i}}+\left(\mathfrak{e}_{i} \mathfrak{i}_{j}+\mathfrak{i}_{j} \mathfrak{e}_{i}\right) \phi_{; i} \phi_{; j} \\
= & \Delta_{g}+\left(\mathfrak{e}_{i} \mathfrak{i}_{j}+\mathfrak{i}_{j} \mathfrak{e}_{i}-\mathfrak{i}_{i} \mathfrak{e}_{j}-\mathfrak{e}_{j} \mathfrak{i}_{i}\right) \phi_{; j} \nabla_{e_{i}}+\left(\mathfrak{e}_{i} \mathfrak{i}_{j}-\mathfrak{i}_{i} \mathfrak{e}_{j}\right) \phi_{; j i}+\phi_{; i} \phi_{; i} \\
= & \Delta_{g}+\left(\mathfrak{e}_{i} \mathfrak{i}_{j}-\mathfrak{i}_{i} \mathfrak{e}_{j}\right) \phi_{; j i}+\phi_{; i} \phi_{; i} .
\end{aligned}
$$

This shows that the associated connection does not depend on $\phi$ and hence is the Levi-Civita connection [12. Since the standard Weitzenböck formulas yield $E\left(\Delta_{g}\right)=-\frac{1}{2} \gamma_{i} \gamma_{j} \Omega_{i j}$, Assertion (3) follows.

We refer to [6] for the proof of Assertion (4). Let $\omega_{+}:=e^{a_{1}} \wedge \ldots \wedge e^{a_{\ell}}$ and let $\omega_{-}:=e^{m} \wedge \omega_{+}$. We then have $\chi \omega_{ \pm}= \pm \omega_{ \pm}$. We use Assertion (4) to prove Assertion (5) by computing:

$$
\begin{aligned}
\left(\nabla_{e_{a}} \chi-\chi \nabla_{e_{a}}\right) \omega_{+} & =\left(\Gamma_{a b c} \mathfrak{e}_{c} \mathfrak{i}_{b}+\Gamma_{a b m} \mathfrak{e}_{m} \mathfrak{i}_{b}-\Gamma_{a b c} \mathfrak{e}_{c} \mathfrak{i}_{b}+\Gamma_{a b m} \mathfrak{e}_{m} \mathfrak{i}_{b}\right) \omega_{+} \\
& =2 L_{a b} \mathfrak{e}_{m} \mathfrak{i}_{b} \omega_{+}, \\
\left(\nabla_{e_{a}} \chi-\chi \nabla_{e_{a}}\right) \omega_{-} & =\left(-\Gamma_{a b c} \mathfrak{e}_{c} \dot{i}_{b}-\Gamma_{a m b} \mathfrak{e}_{b} \mathfrak{i}_{m}+\Gamma_{a b c} \mathfrak{e}_{c} \mathfrak{i}_{b}-\Gamma_{a m b} \mathfrak{e}_{b} \mathfrak{i}_{m}\right) \omega_{-} \\
& =2 L_{a b} \mathfrak{e}_{b} \mathfrak{i}_{m} \omega_{-} .
\end{aligned}
$$

We now discuss functorial properties of the supertrace asymptotics.

Lemma 2.3. (1) On the circle, $a_{2,1}^{d+\delta}=\frac{1}{\sqrt{\pi}} \phi_{; 11}$.

(2) We have $a_{n, m}^{d+\delta}(\phi, g)(x)=(-1)^{m} a_{n, m}^{d+\delta}(-\phi, g)(x)$.

(3) We have $\int_{\partial M} a_{m+1, m, 0}^{d+\delta}(0, g) d y=0$.

(4) Let $(M, \phi, g):=\left(M_{1} \times M_{2}, \phi_{1}+\phi_{2}, g_{1}+g_{2}\right)$ where $\partial M_{1}=\emptyset$. Then

(a) $a_{n, m}^{d+\delta}(\phi, g)=\sum_{n_{1}+n_{2}=n} a_{n_{1}, m_{1}}^{d+\delta}\left(\phi_{1}, g_{1}\right) \cdot a_{n_{2}, m_{2}}^{d+\delta}\left(\phi_{2}, g_{2}\right)$, 
(b) $a_{n, m, k}^{d+\delta}(\phi, g)=\sum_{n_{1}+n_{2}=n} a_{n_{1}, m_{1}}^{d+\delta}\left(\phi_{1}, g_{1}\right) \cdot a_{n_{2}, m_{2}, k}^{d+\delta}\left(\phi_{2}, g_{2}\right)$.

Proof. Asssertion (1) follows from Lemma 2.1 (3) and from Lemma 2.2 (3).

Since the interior invariants $a_{n, m}^{d+\delta}(\phi, g)$ are local, we may suppose without loss of generality that $M$ is a closed orientable manifold in the proof of Assertion (2). Let $\tilde{\star}_{g}$ be the normalized Hodge operator defined by the metric. Then, the normalizations having taken into account the sign conventions, the usual intertwining relations extend to the twisted context to show

$$
\tilde{\star}_{g}^{2}=\mathrm{id}, \quad \tilde{\star}_{g} d_{\phi} \tilde{\star}_{g}=\delta_{-\phi, g}, \quad \text { and } \quad \tilde{\star}_{g} \delta_{\phi, g} \tilde{\star}_{g}=d_{-\phi} .
$$

Assertion (2) now follows from the intertwining relationship:

$$
\tilde{\star}_{g} \Delta_{\phi, g}^{p} \tilde{\star}_{g}=\Delta_{-\phi, g}^{m-p} .
$$

We note that $\tilde{\star}_{g}$ intertwines absolute and relative boundary conditions; thus we can not conclude a similar equivariance property for the boundary invariants.

We use Theorem 1.1 to see that $a_{m+1, m}^{d+\delta}(0, g)=0$ regardless of the parity of $m$. As the interior invariant vanishes pointwise, the boundary integral vanishes by Equation (1.a).

To prove Assertion (4), we decompose

$$
\Lambda(M)=\Lambda\left(M_{1}\right) \otimes \Lambda\left(M_{2}\right), d_{\phi}=d_{1}+d_{2}, \text { and } \delta_{\phi, g}=\delta_{1}+\delta_{2}
$$

where, on $C^{\infty}\left(\Lambda^{p}\left(M_{1}\right) \otimes \Lambda^{q}\left(M_{2}\right)\right)$, we have

$$
\begin{array}{ll}
d_{1}:=d_{\phi_{1}} \otimes \mathrm{Id}, & d_{2}:=(-1)^{p} \mathrm{Id} \otimes d_{\phi_{2}}, \\
\delta_{1}:=\delta_{\phi_{1}, g_{1}} \otimes \mathrm{Id}, & \delta_{2}:=(-1)^{p} \mathrm{Id} \otimes \delta_{\phi_{2}, g_{2}} .
\end{array}
$$

Consequently these operators satisfy the commutation relations:

$$
d_{1} d_{2}+d_{2} d_{1}=0, d_{1} \delta_{2}+\delta_{2} d_{1}=0, \delta_{1} d_{2}+d_{2} \delta_{1}=0, \delta_{1} \delta_{2}+\delta_{2} \delta_{1}=0 .
$$

Thus the associated Laplacian and fundamental solution of the heat equation decompose in the form

$$
\begin{aligned}
& \Delta_{\phi, g}^{p}=\oplus_{p=p_{1}+p_{2}} \Delta_{\phi_{1}, g_{1}}^{p_{1}} \otimes \mathrm{Id}+\mathrm{Id} \otimes \Delta_{\phi_{2}, g_{2}}^{p_{2}}, \\
& e^{-t \Delta_{\phi, g, \mathcal{B}_{a}}^{p}}=\oplus_{p=p_{1}+p_{2}} e^{-t \Delta_{\phi_{1}, g_{1}}^{p_{1}}} \otimes e^{-t \Delta_{\phi_{2}, g_{2}, \mathcal{B}_{a}}^{p_{2}}} .
\end{aligned}
$$

Let $f=f_{1} f_{2}$ where $f_{i} \in C^{\infty}\left(M_{i}\right)$. We then have

$$
\operatorname{Tr}_{L^{2}}\left\{f e^{-t \Delta_{\phi, g, \mathcal{B}_{a}}^{p}}\right\}=\sum_{p=p_{1}+p_{2}} \operatorname{Tr}_{L^{2}}\left\{f_{1} e^{-t \Delta_{\phi_{1}, g_{1}}^{p_{1}}}\right\} \cdot \operatorname{Tr}_{L^{2}}\left\{f_{2} e^{-t \Delta_{\phi_{2}, g_{2}, \mathcal{B}_{a}}^{p_{2}}}\right\}
$$

Assertion (4) now follows by equating coefficients in the asymptotic expansion of the supertrace.

\section{INVARIANCE THEORY}

Let $V$ be an $m$ dimensional real vector space which is equipped with a positive definite inner product $g(\cdot, \cdot)$. Let $O(V)$ be the associated orthogonal group. One says that a polynomial map $f: \times^{k} V \rightarrow \mathbb{R}$ is an orthogonal invariant if

$$
f\left(\xi v^{1}, \ldots, \xi v^{k}\right)=f\left(v^{1}, \ldots, v^{k}\right) \quad \forall \xi \in O(V) \quad \text { and } \quad \forall\left(v^{1}, \ldots, v^{k}\right) \in \times{ }^{k} V .
$$

Weyl's first theorem of invariants [22] (Theorem 2.9.A) is the following:

Theorem 3.1. Every orthogonal invariant depending on $k$ vectors $\left(v_{1}, \ldots, v_{k}\right)$ in $\times{ }^{k} V$ is expressible in terms of the $k^{2}$ scalar invariants $g\left(v_{i}, v_{j}\right)$. 
Let $\mathcal{I}_{k, m}$ be the set of all multilinear invariant maps from $\times{ }^{k} V$ to $\mathbb{R}$; only the dimension $m$ of $V$ is really relevant so we suppress $V$ from the notation. Given our interest is in $O(V)$ and not $S O(V)$ invariance, we have $\mathcal{I}_{k, m}=\{0\}$ if $k$ is odd. Consequently, we shall suppose that $k$ is even henceforth. Let $\Sigma_{k}$ be the group of all permutations of the set $\{1, \ldots, k\}$. We define a multi-linear invariant map $p_{k, \sigma}$ for any permutation $\sigma \in \Sigma_{k}$ by setting:

$$
p_{k, \sigma}\left(v_{1}, \ldots, v_{k}\right):=g\left(v_{\sigma(1)}, v_{\sigma(2)}\right) \cdots g\left(v_{\sigma(k-1)}, v_{\sigma(k)}\right) .
$$

Theorem 3.2. $\mathcal{I}_{k, m}=\operatorname{span}_{\sigma \in \Sigma_{k}}\left\{p_{k, \sigma}\right\}$.

Proof. We use Theorem 3.1 to express $p \in \mathcal{I}_{k, m}$ in terms of monomials involving the inner products $g\left(v_{i}, v_{j}\right)$. Since $p$ is multi-linear,

$$
p\left(c v_{1}, v_{2}, \ldots, v_{k}\right)=c p\left(v_{1}, v_{2}, \ldots, v_{k}\right) .
$$

Consequently we need only consider monomials where the variable $v_{1}$ appears exactly once as otherwise we contradict multi-linearity. A similar observation holds for the remaining indices and these are exactly the expressions $p_{k, \sigma}$ defined above.

In view of Theorem 3.2 one says 'invariant multilinear maps are given by contractions of indices' as, relative to an orthonormal basis, the inner products involved correspond to contraction of indices in pairs. Let $\left\{e_{i}\right\}$ be an orthonormal basis for the vector space $V$ and let $\omega=\omega_{i_{1} i_{2} \ldots i_{k}} e_{i_{1}} \otimes \ldots \otimes e_{i_{k}} \in \otimes^{k} V$. We have, for example:

$$
\begin{aligned}
& \mathcal{I}_{2, m}:=\operatorname{Span}\left\{\omega \rightarrow \omega_{i i}\right\}, \quad \text { and } \\
& \mathcal{I}_{4, m}:=\operatorname{Span}\left\{\omega \rightarrow \omega_{i i j j}, \omega \rightarrow \omega_{i j i j}, \omega \rightarrow \omega_{i j j i}\right\} .
\end{aligned}
$$

Let $\mathcal{P}_{n, m}$ be the space of invariant polynomials which are homogeneous of weight $n$ in the derivatives of the metric tensor. Atiyah, Bott, and Patodi 2 applied this formalism to study these spaces. In geodesic coordinate systems, all jets of the metric can be computed in terms of the covariant derivatives of the curvature tensor and vice versa. Thus, for example, if $n=4$, an invariant $P \in \mathcal{P}_{4, m}$ can be regarded as a map from a certain subspace

$$
W \subset\left\{\otimes^{6} T(M)\right\} \oplus\left\{\otimes^{8} T(M)\right\}
$$

to $\mathbb{R}$ which is invariant under the action of the orthogonal group; here $W$ is generated by the algebraic covariant derivatives $\nabla^{2} R \subset \otimes^{6} T(M)$ and by the algebraic curvature tensors $R \otimes R \subset \otimes^{8} T(M)$. As the subspace $W$ is orthogonally invariant, extending $P$ to be zero on $W^{\perp}$ defines an orthogonally invariant map to which Theorem 3.2 applies. Thus, for example, after taking into account the appropriate curvature symmetries, one has:

$$
\begin{aligned}
& \mathcal{P}_{2, m}=\operatorname{Span}\left\{\tau:=R_{i j j i}\right\} \\
& \mathcal{P}_{4, m}=\operatorname{Span}\left\{\tau^{2},\left|\rho^{2}\right|:=R_{i j j k} R_{i l l k},|R|^{2}:=R_{i j k l} R_{i j k l}, \Delta \tau:=-R_{i j j i ; k k}\right\}
\end{aligned}
$$

This analysis extends to form valued invariants with coefficients in an auxiliary vector bundle and gives rise to a heat equation proof of the index theorem for the classical elliptic complexes [2].

What is relevant to our analysis, however, is Weyl's second main theorem 22 ] (Theorem 2.17.A). 
Theorem 3.3. Every relation among scalar products is an algebraic consequence of the relation

$$
0=\operatorname{det}\left(\begin{array}{llll}
g\left(v_{1}, w_{1}\right) & g\left(v_{2}, w_{1}\right) & \ldots & g\left(v_{m+1}, w_{1}\right) \\
g\left(v_{1}, w_{2}\right) & g\left(v_{2}, w_{2}\right) & \ldots & g\left(v_{m+1}, w_{2}\right) \\
\ldots & \ldots & \ldots & \ldots \\
g\left(v_{1}, w_{m+1}\right) & g\left(v_{2}, w_{m+1}\right) & \ldots & g\left(v_{m+1}, w_{m+1}\right)
\end{array}\right) .
$$

We remark that this relation can also be expressed in the form:

$$
0=g\left(v_{1} \wedge \ldots \wedge v_{m+1}, w_{1} \wedge \ldots \wedge w_{m+1}\right) .
$$

Let $W$ be a vector space of dimension $m-1$. Choose an inner product preserving inclusion $i: W \subset V$ which embeds $O(W) \subset O(V)$. We define the restriction map

$$
r: \mathcal{I}_{k, m} \rightarrow \mathcal{I}_{k, m-1}
$$

which is characterized dually by the property:

$$
r(p)\left(w_{1}, \ldots, w_{k}\right)=p\left(i\left(w_{1}\right), \ldots, i\left(w_{k}\right)\right) .
$$

If $p$ is given by contractions of indices which range from 1 to $m$, then $r(p)$ is given by restricting the range of summation to range from 1 to $m-1$. Consequently, the map $r$ is surjective. If $k \geq 2 m$ and if $\sigma \in \Sigma_{k}$, define:

$$
\begin{aligned}
\Theta_{k, m, \sigma}\left(v_{1}, \ldots, v_{k}\right):= & g\left(v_{\sigma(1)} \wedge \ldots \wedge v_{\sigma(m)}, v_{\sigma(m+1)} \wedge \ldots \wedge v_{\sigma(2 m)}\right) \\
& \times g\left(v_{\sigma(2 m+1)}, v_{\sigma(2 m+2)}\right) \cdots g\left(v_{\sigma(k-1)}, v_{\sigma(k)}\right) .
\end{aligned}
$$

Theorem 3.4. Let $m \geq 2$.

(1) $r: \mathcal{I}_{k, m} \rightarrow \mathcal{I}_{k, m-1}$ is surjective.

(2) $r: \mathcal{I}_{k, m} \rightarrow \mathcal{I}_{k, m-1}$ is injective if $k<2 m$.

(3) If $k \geq 2 m$, then $\operatorname{ker}(r) \cap \mathcal{I}_{k, m}=\operatorname{span}_{\sigma \in \Sigma_{k}}\left\{\Theta_{k, m, \sigma}\right\}$.

Proof. We have already verified Assertion (1). To prove Assertion (2), we use Theorem 3.2 to express $p \in \mathcal{I}_{k, m}$ in terms of inner products. We use Theorem 3.3. after making an appropriate dimension shift, to see that $r(p)$ vanishes if and only if it can be written as sums of terms each of which is divisible by an appropriate determinant $J$ of size $m \times m$. The desired result now follows from equation (3.a) and from the same arguments used to prove Theorem 3.2

Previously we have considered invariants of the metric alone. The analysis extends easily to the twisted setting. We define

$$
\operatorname{weight}\left(\nabla^{k} \phi\right)=k \quad \text { and } \quad \operatorname{weight}\left(\nabla^{k} R\right)=2+k .
$$

Let $\mathcal{Q}_{n, m}$ be the space of all $O(m)$ invariant polynomials of total weight $n$ in the components of $R$, the covariant derivatives of $R$, and the covariant derivatives of $\phi$. We do not admit $\phi$ as a variable. Furthermore we require that each monomial either does not involve the covariant derivatives of $\phi$ at all or involves at least two covariant derivatives of $\phi$. We use the $\mathbb{Z}_{2}$ action $\phi \rightarrow-\phi$ to decompose

$$
\begin{aligned}
& \mathcal{Q}_{n, m}=\mathcal{Q}_{n, m}^{+} \oplus \mathcal{Q}_{n, m}^{-} \text {where } \\
& \mathcal{Q}_{n, m}^{ \pm}:=\left\{Q \in \mathcal{Q}_{n, m}: Q(\phi, g)= \pm Q(-\phi, g)\right\} .
\end{aligned}
$$

The restriction map in Theorem 3.4 induces natural surjective maps

$$
r: \mathcal{Q}_{n, m}^{ \pm} \rightarrow \mathcal{Q}_{n, m-1}^{ \pm} \rightarrow 0 .
$$


If $\left(N, \phi_{N}, g_{N}\right)$ are structures in dimension $m-1$, then we can define corresponding structures in dimension $m$ by setting

$$
\left(M, \phi_{M}, g_{M}\right):=\left(N \times S^{1}, \phi_{N}, g_{N}+d \theta^{2}\right) .
$$

If $y \in \partial N$ is the point of evaluation, let $(y, 1) \in \partial M$ be the corresponding point of evaluation - it does not matter which point is chosen on the circle owing to the rotational symmetry. The restriction map $r: \mathcal{Q}_{n, m} \rightarrow \mathcal{Q}_{n, m-1}$ is then characterized dually by the formula:

$$
r(Q)\left(\phi_{N}, g_{N}\right)(y)=Q\left(\phi_{N}, g_{N}+d \theta^{2}\right)(y, 1) .
$$

Lemma 3.5. (1) If $m$ is even, then $a_{n, m}^{d+\delta}(\phi, g) \in \mathcal{Q}_{n, m}^{+} \cap \operatorname{ker} r$.

(2) If $m$ is odd, then $a_{n, m}^{d+\delta}(\phi, g) \in \mathcal{Q}_{n, m}^{-} \cap \operatorname{ker} r$.

Proof. Standard arguments [12] show the invariants $a_{n, m}^{d+\delta}(\phi, g)$ are homogeneous of weight $n$ in the jets of the metric and of $\phi$. Let $\nabla$ be the Levi-Civita connection on $\Lambda M$. By Lemma 2.2 (1),

$$
\Delta_{\phi, g}^{p}=\Delta_{g}+\frac{1}{2} \gamma_{i} \gamma_{j} \Omega_{i j}+\phi_{; i} \phi_{; i}-\phi_{; j i}\left(\mathfrak{e}_{i} \mathfrak{i}_{j}-\mathfrak{i}_{j} \mathfrak{e}_{i}\right) .
$$

Thus the undifferentiated variable $\phi$ does not play a role in these invariants. Furthermore, either at least 2 covariant derivatives of $\phi$ appear or only the curvature $R$ appears in each Weyl monomial of $a_{n, m}^{d+\delta}(\phi, g)$. This shows that

$$
a_{n, m}^{d+\delta}(\phi, g) \in \mathcal{Q}_{n, m}
$$

We use Lemma 2.3 (2) to see that $a_{n, m}^{d+\delta}(\phi, g)$ is an odd function of $\phi$ if $m$ is odd and an even function of $\phi$ if $m$ is even. To complete the proof, we must show $r a_{n, m}^{d+\delta}=0$. Suppose that $M=N \times S^{1}$ has the product metric and that $\phi=\phi_{N}$ is independent of the angular parameter $\theta \in S^{1}$. As $\phi_{S^{1}}=0$, we use Lemma 2.3 (3) to see $a_{n, 1}^{d+\delta}\left(0, g_{S^{1}}\right)=(-1)^{1} a_{n, 1}^{d+\delta}\left(0, g_{S^{1}}\right)=0$ for all $n$. Thus Lemma 2.3 (4a) implies that $a_{n, m}^{d+\delta}\left(\phi_{M}, g_{N}\right)=0$. This shows that $r a_{n, m}^{d+\delta}=0$.

Assertion (1) of Lemma 1.3 will follow from the following result:

Lemma 3.6. If $m$ is odd, then $\mathcal{Q}_{m+1, m}^{-} \cap \operatorname{ker} r=\operatorname{Span}\left\{\mathcal{E}_{m+1, m}\right\}$.

Proof. Let $0 \neq Q \in \mathcal{Q}_{m+1, m}^{-}$. Let $A$ be a monomial of $Q$ of the form

$$
A=\phi_{; \alpha_{1}} \ldots \phi_{; \alpha_{u}} R_{i_{1} j_{1} k_{1} \ell_{1} ; \beta_{1}} \ldots R_{i_{v} j_{v} k_{v} \ell_{v} ; \beta_{v}}
$$

where $\alpha_{\mu}$ and $\beta_{\nu}$ denote appropriate collections of indices. Then

$$
m+1=\operatorname{weight}(A)=\sum_{\mu}\left|\alpha_{\mu}\right|+\sum_{\nu}\left(2+\left|\beta_{\nu}\right|\right) .
$$

By definition, the empty sum is 0 . Thus $\sum_{\mu}$ is to be ignored if $u=0$ and $\sum_{\nu}$ is to be ignored if $v=0$. Let $k$ be total number of indices present in $A$;

$$
k:=\sum_{\mu}\left|\alpha_{\mu}\right|+\sum_{\nu}\left(4+\left|\beta_{\nu}\right|\right)=\operatorname{weight}(A)+2 v=m+1+2 v .
$$

We apply Weyl's second main theorem of invariance theory as discussed above. To ensure that $r Q=0$, we must contract $2 m$ indices in $A$ using the $\varepsilon$ tensor and then contract the remaining indices of $A$ in pairs. Consequently, at least $2 m$ indices must appear in $A$ so

$$
2 m \leq k=m+1+2 v=2 m+2-\sum_{\mu}\left|\alpha_{\mu}\right|-\sum_{\nu}\left|\beta_{\nu}\right| \leq 2 m+2 .
$$


Since $m$ is odd, $2 m, m+1+2 v$, and $2 m+2$ are all even. Thus only one of the two inequalities given in Display (3.b) can be strict. As $Q(-\phi, g)=-Q(\phi, g), u$ must be odd. Thus

$$
\sum_{\mu}\left|\alpha_{\mu}\right|>0
$$

so the second inequality in Equation (3.b) is strict. Thus exactly $2 m=k$ indices appear in $A$ and all are contracted using the $\varepsilon$ tensor. The first and second Bianchi identity show $R_{* * * * ; \beta}=0$ if 3 indices are alternated. Thus at most two $i$ indices and at most two $j$ indices can appear in each $R_{* * * * ;}$ variable. This shows that

$$
\left|\beta_{\nu}\right|=0 \text { for all } \nu \text {. }
$$

Furthermore, the two possibilities are $R_{i_{1} i_{2} j_{2} j_{1}}$ or $R_{i_{1} j_{1} i_{2} j_{2}}$. The first Bianchi identity can then be used to express the second variable in terms of the first. Since

$$
u \leq\left|\alpha_{1}\right|+\ldots+\left|\alpha_{u}\right|=2
$$

and $u$ is odd, $u=1$ and $\left|\alpha_{1}\right|=2$, since either 0 or at least 2 covariant derivatives of $\phi$ appear in each monomial of $Q \in \mathcal{Q}_{n, m}$. Thus we are in fact dealing with a multiple of $\mathcal{E}_{m+1, m}:=\varepsilon_{J}^{I} \phi_{; i_{1} j_{1}} \mathcal{R}_{J, 2}^{I, m}$.

To complete the proof of Lemma 1.3 we study the boundary invariants. Let $\tilde{\nabla}$ denote the Levi-Civita connection of the boundary. We consider polynomials in the components of the tensors

$$
\left\{R, \nabla R, \nabla^{2} R, \ldots, L, \tilde{\nabla} L, \tilde{\nabla}^{2} L, \ldots, \nabla \phi, \nabla^{2} \phi, \ldots\right\} .
$$

Again, we do not introduce the variable $\phi$. We let

$$
\text { weight }\left(\nabla^{k} R\right):=2+k, \operatorname{weight}\left(\tilde{\nabla}^{k} L\right):=1+k, \text { and weight }\left(\nabla^{k} \phi\right)=k .
$$

Let $\tilde{\mathcal{Q}}_{n, m}$ be the space of all $O(m-1)$ invariant polynomials of total weight $n$ where we admit monomials which either do not involve the covariant derivatives of $\phi$ at all or which involve at least two covariant derivatives of $\phi$.

Let $\tilde{\mathcal{P}}_{n, m} \subset \tilde{\mathcal{Q}}_{n, m}$ be the subspace of invariants which do not involve the covariant derivatives of $\phi$. Setting $\phi=0$ defines a natural map from $\tilde{\mathcal{Q}}_{n, m}$ to $\tilde{\mathcal{P}}_{n, m}$. If $P \in \tilde{\mathcal{P}}_{n, m}$, then the evaluation $\mathfrak{I}(P)(g) \in \mathbb{R}$ is defined by setting

$$
\Im P(g):=\int_{\partial M} P(g)(y) d y .
$$

By Lemma $2.3(3), \Im a_{m+1, m, 0}^{d+\delta}(0, g)=0$. The same argument as that given to establish Lemma 3.5 can be used to show that $a_{n, m, k}^{d+\delta} \in \tilde{\mathcal{Q}}_{n-k-1} \cap \operatorname{ker} r$. The remaining assertions of Lemma 1.3 will now follow from the following result:

Lemma 3.7. (1) $\tilde{\mathcal{Q}}_{n, m} \cap \operatorname{ker} r=\{0\}$ if $n<m-1$.

(2) $\tilde{\mathcal{Q}}_{m-1, m} \cap \operatorname{ker} r=\operatorname{Span}_{k}\left\{\mathcal{F}_{m-1, m}^{k}\right\}$.

(3) $\tilde{\mathcal{Q}}_{m, m} \cap \operatorname{ker} r=\operatorname{Span}_{k}\left\{\mathcal{F}_{m, m}^{1, k}, \mathcal{F}_{m, m}^{2, k}\right\}+\left\{\tilde{\mathcal{P}}_{m, m} \cap \operatorname{ker} r\right\}$.

(4) $\tilde{\mathcal{P}}_{m, m} \cap \operatorname{ker} r \cap \operatorname{ker} \mathfrak{I}=\operatorname{Span}_{k}\left\{\mathcal{F}_{m, m}^{3, k}\right\}$.

Proof. Let $0 \neq Q \in \tilde{\mathcal{Q}}_{n, m} \cap \operatorname{ker} r$ and let $A$ be a monomial of $Q$ of weight $n$ where:

$$
\begin{aligned}
& A:=\phi_{; \alpha_{1}} \cdots \phi_{; \alpha_{u}} R_{i_{1} j_{1} k_{1} \ell_{1} ; \beta_{1}} \cdots R_{i_{v} j_{v} k_{v} \ell_{v} ; \beta_{v}} L_{a_{1} b_{1}: \gamma_{1}} \cdots L_{a_{w} b_{w}: \gamma_{w}}, \\
& n:=\sum_{\mu}\left|\alpha_{\mu}\right|+\sum_{\nu}\left(\left|\beta_{\nu}\right|+2\right)+\sum_{\sigma}\left(\left|\gamma_{\sigma}\right|+1\right) .
\end{aligned}
$$

To ensure that $r Q=0$, we contract $2(m-1)$ tangential indices in $A$ using the $\varepsilon$ tensor; the remaining tangential indices must be contracted in pairs. Since the 
structure group is $O(m-1)$, the normal index ' $m$ ' can stand alone and unchanged. Let $k_{T}$ be the total number of tangential indices in $A$, and let $k_{m}$ be the total number of times the normal index $m$ appears in $A$. We estimate:

$$
\begin{aligned}
& 2 m-2 \leq k_{T} \leq k_{T}+k_{m} \\
& =\sum_{\mu}\left|\alpha_{\mu}\right|+\sum_{\nu}\left(\left|\beta_{\nu}\right|+4\right)+\sum_{\sigma}\left(\left|\gamma_{\sigma}\right|+2\right)=n+2 v+w \\
& =2 n-\sum_{\mu}\left|\alpha_{\mu}\right|-\sum_{\nu}\left|\beta_{\nu}\right|-\sum_{\sigma}\left|\gamma_{\sigma}\right| \leq 2 n .
\end{aligned}
$$

Assertion (1) of the Lemma follows as this is not possible if $n<m-1$.

We set $n=m-1$ to prove Assertion (2). All the inequalities of Display (3.C) must have been equalities so there are no covariant derivatives and thus the $\phi$ variables do not appear. All the indices are tangential and are contracted using the $\varepsilon$ tensor. After using the first Bianchi identity, we see that this leads to the invariants $\mathcal{F}_{m-1, m}^{k}$ which proves Assertion (2).

Let $n=m$. Display (3.C) involves a total increase of 2. Thus at most 2 explicit covariant derivatives are present. However, unless at least 2 covariant derivatives are present, $\phi$ is not involved and this leads to invariants in $\tilde{\mathcal{P}}_{m, m} \cap \operatorname{ker} r$. Thus we may suppose exactly 2 explicit covariant derivatives are present - and all of them appear on $\phi$. Consequently

$$
k_{T}=2 m-2, k_{m}=0, \sum_{\mu}\left|\alpha_{\mu}\right|=2, \sum_{\nu}\left|\beta_{\nu}\right|=0 \text {, and } \sum_{\sigma}\left|\gamma_{\sigma}\right|=0 .
$$

Since every index is tangential and all are contracted using the tensor $\varepsilon$, after applying the Bianchi identities, we obtain the invariants $\mathcal{F}_{m, m}^{1, k}$ and $\mathcal{F}_{m, m}^{2, k}$. This completes the proof of Assertion (3).

To prove Assertion (4), we set $\phi=0$ and consider only metric invariants. Let $\tilde{\mathcal{P}}_{n, m}^{p}$ be the space of $p$ form valued invariants which are homogeneous of degree $n$ in the derivatives of the metric; $\tilde{\mathcal{P}}_{n, m}=\tilde{\mathcal{P}}_{n, m}^{0}$.

Let $\tilde{\delta}: \tilde{\mathcal{P}}_{n, m}^{p} \rightarrow \tilde{\mathcal{P}}_{n+1, m}^{p-1}$ be the coderivative of the boundary. Results of [1] describe the cohomology groups of this complex. When combined with standard methods of invariance theory they yield the following observations:

(1) $r$ is a surjective map from $\tilde{\mathcal{P}}_{n, m}^{p}$ to $\tilde{\mathcal{P}}_{n, m-1}^{p}$ with $r \tilde{\delta}=\tilde{\delta} r$.

(2) If $n \neq m-1$, then $\tilde{\mathcal{P}}_{n, m}^{0} \cap \operatorname{ker} \mathfrak{I}=\tilde{\delta} \tilde{\mathcal{P}}_{n-1, m}^{1}$.

(3) If $n \neq m-1$, then $\tilde{\mathcal{P}}_{n-1, m}^{1} \cap \operatorname{ker} \tilde{\delta}=\tilde{\delta} \tilde{\mathcal{P}}_{n-2, m}^{2}$.

Let $P_{m, m} \in \tilde{\mathcal{P}}_{m, m} \cap$ ker $r \cap$ ker $\mathfrak{I}$. Choose $P_{m-1, m}^{1} \in \tilde{\mathcal{P}}_{m-1, m}^{1}$ so $\tilde{\delta} P_{m-1, m}^{1}=P_{m, m}$. Unfortunately, $r P_{m-1, m}^{1}$ need not vanish and we must adjust $P_{m-1, m}^{1}$. Since

$$
\tilde{\delta} r P_{m-1, m}^{1}=r \tilde{\delta} P_{m-1, m}^{1}=r P_{m, m}=0,
$$

we may choose $P_{m-2, m-1}^{2} \in \tilde{\mathcal{P}}_{m-2, m-1}^{2}$ so $\tilde{\delta} P_{m-2, m-1}^{2}=r P_{m-1, m}^{1}$. Since $r$ is surjective, we may choose $P_{m-2, m}^{2} \in \tilde{\mathcal{P}}_{m-2, m}^{2}$ so $r P_{m-2, m}^{2}=P_{m-2, m-1}^{2}$. Then:

$$
\begin{aligned}
& \tilde{\delta}\left\{P_{m-1, m}^{1}-\tilde{\delta} P_{m-2, m}^{2}\right\}=\tilde{\delta} P_{m-1, m}^{1}=P_{m, m}, \\
& r\left\{P_{m-1, m}^{1}-\tilde{\delta} P_{m-2, m}^{2}\right\}=r P_{m-1, m}^{1}-\tilde{\delta} r P_{m-2, m}^{2} \\
& \quad=r P_{m-1, m}^{1}-\tilde{\delta} P_{m-2, m-1}^{2}=0 .
\end{aligned}
$$

Consequently

$$
\tilde{\mathcal{P}}_{m, m} \cap \operatorname{ker} r \cap \operatorname{ker} \mathfrak{I}=\tilde{\delta}\left\{\tilde{\mathcal{P}}_{m-1, m}^{1} \cap \operatorname{ker} r\right\} .
$$


Let $0 \neq P_{m-1, m}^{1} \in \tilde{\mathcal{P}}_{m-1, m}^{1} \cap \operatorname{ker} r$ and let

$$
A=R_{i_{1} j_{1} k_{1} \ell_{1} ; \beta_{1}} \ldots R_{i_{v} j_{v} k_{v} \ell_{v} ; \beta_{v}} L_{a_{1} b_{1}: \gamma_{1}} \ldots L_{a_{w} b_{w}: \gamma_{w}} e^{c}
$$

be a monomial of $P_{m-1, m}^{1}$. Since $r P_{m-1, m}^{1}=0$, we must contract $2(m-1)$ indices in $A$ using the $\varepsilon$ tensor and contract the remaining indices in pairs. We estimate

$$
\begin{aligned}
& 2(m-1) \leq k_{T} \leq k_{T}+k_{m}=\sum_{\nu}\left(\left|\beta_{\nu}\right|+4\right)+\sum_{\sigma}\left(\left|\gamma_{\sigma}\right|+2\right)+1 \\
& =m-1+2 v+w+1=2(m-1)-\sum_{\nu}\left|\beta_{\nu}\right|-\sum_{\sigma}\left|\gamma_{\sigma}\right|+1 \\
& \leq 2(m-1)+1 .
\end{aligned}
$$

This sequence of inequalities represents a total increase of 1 . Thus $k_{T}=2(m-1)$ and every tangential index is contracted using the $\varepsilon$ tensor. We have

$$
L_{c_{2} c_{3}: c_{1}}-L_{c_{1} c_{3}: c_{2}}=R_{c_{1} c_{2} c_{3} m} .
$$

We may therefore assume $\left|\gamma_{\sigma}\right|=0$ so there are no tangential derivatives of $L$ present. If $k_{m}=0$, then every index is contracted using the $\varepsilon$ tensor. Thus the Bianchi identities show $\left|\beta_{\nu}\right|=0$ for all $\nu$. This means that every inequality in Display (3.e) is an equality which is impossible. Consequently $k_{m}=1$ and $\sum_{\nu}\left|\beta_{\nu}\right|=0$. This leads to the invariants

$$
\mathcal{G}_{m-1, m}^{k}:=\varepsilon_{A}^{B} \mathcal{R}_{B, 1}^{A, 2 k} R_{a_{2 k+1} a_{2 k+2} m b_{2 k+1}} \mathcal{L}_{B, 2 k+3}^{A, m-1} e^{b_{2 k+2}} .
$$

Assertion (4) now follows from Equation (3.d) since $\tilde{\delta} \mathcal{G}_{m-1, m}^{k}=-\mathcal{F}_{m, m}^{3, k}$.

\section{Product formulas, special case computations, and functorial PROPERTIES}

Throughout this section, we adopt the notation of Lemma 1.3 We begin with a result which is based on product formulas.

Lemma 4.1.

(1) If $m=2 \bar{m}+1$, then $c_{m+1, m}=\frac{1}{\sqrt{\pi} 8^{\bar{m}} \pi^{\bar{m}} \bar{m} !}$

(2) If $k>0$, then $c_{m+1, m, 1}^{k}=\frac{1}{\pi^{k} 8^{k} k !} c_{m-2 k+1, m-2 k, 1}^{0}$.

(3) If $k>0$, then $c_{m+1, m, 0}^{i, k}=\frac{1}{\pi^{k} 8^{k} k !} c_{m-2 k+1, m-2 k, 0}^{i, 0}$.

(4) We have $c_{m+1, m, 0}^{1,0}=\frac{1}{\sqrt{\pi}} \frac{1}{(m-2) ! \operatorname{vol}\left(S^{m-2}\right)}$ and $c_{m+1, m, 0}^{2,0}=0$.

Proof. Give $S^{m}$ and $D^{m}$ the standard metrics $g_{S, m}$ and $g_{D, m}$. We then have

$$
\varepsilon_{J}^{I} \mathcal{R}_{J, 1}^{I, 2 \bar{m}}\left(g_{S, m}\right)=2^{\bar{m}}(2 \bar{m}) ! \quad \text { and } \quad \varepsilon_{A}^{B} \mathcal{L}_{A, 1}^{B, m-1}\left(g_{D, m}\right)=(m-1) ! .
$$

Let $m=2 \bar{m}+1$. Give $M:=S^{1} \times S^{2 \bar{m}}$ the product structures where $\phi_{2}=0$. By Theorem 1.1 (1) and Lemma 2.3 (4) we have

$$
a_{m+1, m}^{d+\delta}\left(\phi_{M}, g_{M}\right)=a_{2,1}^{d+\delta}\left(\phi_{1}, g_{S, 1}\right) a_{2 \bar{m}, 2 \bar{m}}\left(0, g_{S, 2 \bar{m}}\right) .
$$

Consequently, by Equation (4.a) and by Theorem 1.1](4),

$$
\begin{aligned}
& a_{m+1, m}^{d+\delta}(\phi, g)=c_{m+1, m} \phi_{; 11} 2^{\bar{m}}(2 \bar{m}) ! \\
= & a_{2,1}^{d+\delta}\left(\phi_{1}, g_{S, 1}\right) \cdot a_{2 \bar{m}, 2 \bar{m}}^{d+\delta}\left(0, g_{S, 2 \bar{m}}\right)=\frac{1}{\sqrt{\pi}} \phi_{; 11} \frac{1}{8^{\bar{m}} \pi^{\bar{m}} \bar{m} !} 2^{\bar{m}}(2 \bar{m}) ! .
\end{aligned}
$$

We complete the proof of Asssertion (1) by using this relation to solve for $c_{m+1, m}$ :

$$
c_{m+1, m}=\frac{1}{\sqrt{\pi} 8^{\tilde{m}} \pi^{\tilde{m}} \bar{m} !} .
$$


Fix $k>0$. Give $M=S^{2 k} \times D^{m-2 k}$ the product structures where $\phi_{1}=0$. We argue as in the proof of Assertion (1) to see that:

$$
\begin{aligned}
a_{m+1, m, 1}^{d+\delta}\left(\phi_{M}, g_{M}\right) & =\sum_{j} c_{m+1, m, 1}^{j} \mathcal{F}_{m, m}^{j}\left(\phi_{M}, g_{M}\right) \\
& =c_{m+1, m, 1}^{k} 2^{k}(2 k) ! \cdot(m-2 k-1) ! \\
& =a_{2 k, 2 k}^{d+\delta}\left(0, g_{S, 2 k}\right) \cdot a_{m-2 k+1, m-2 k, 1}^{d+\delta}\left(0, g_{D, m-2 k}\right) \\
& =\frac{1}{\pi^{k} 8^{k} k !} 2^{k}(2 k) ! c_{2 k+1,2 k, 1}^{0}(m-2 k-1) ! .
\end{aligned}
$$

This equation relates $c_{m+1, m, 1}^{k}$ and $c_{m+1, m, 1}^{0}$ and thereby establishes Assertion (2); the proof of Assertion (3) is similar.

Let $M:=S^{1} \times D^{m-1}$ where $\phi=\phi(\theta)$ depends only on $S^{1}$. We use Theorem 1.1 to determine $a_{m-1, m-1,0}^{d+\delta}\left(0, g_{D, m-1}\right)$. As $a_{2,1}^{d+\delta}=\frac{1}{\sqrt{\pi}} \phi_{; 11}$, we argue as above to see

$$
\begin{aligned}
& a_{m+1, m, 0}^{d+\delta}(\phi, g)=\left\{c_{m+1, m, 0}^{1,0} \phi_{; 11}+c_{m+1, m, 0}^{2,0} \phi_{; 1} \phi_{; 1}\right\}(m-2) ! \\
= & a_{2,1}^{d+\delta}\left(\phi, d \theta^{2}\right) \cdot a_{m-1, m-1,0}^{d+\delta}\left(0, g_{D^{m-1}}\right)=\frac{1}{\sqrt{\pi}} \phi_{; 11} \frac{(m-2) !}{\operatorname{vol}\left(S^{m-2}\right)(m-2) !} .
\end{aligned}
$$

We solve for $c_{m+1, m, 0}^{1,0}$ and $c_{m+1, m, 0}^{2,0}$ to establish Assertion (4).

By Lemma 4.1 we need only determine $c_{m+1, m, 1}^{0}$ and $c_{m+1, m, 0}^{3,0}$ to complete the proof of Theorem 1.2 As these terms do not involve $\phi$, we set $\phi=0$ henceforth. We introduce universal constants $\bar{c}_{n, m, k}^{\nu}$ so that if $\mathcal{B}$ defines mixed boundary conditions for an operator of Laplace type, then the heat trace asymptotics have the form:

$$
a_{n, m, k}(y, D, \mathcal{B})=\bar{c}_{n, m, k}^{0} \operatorname{Tr}\left\{S^{n-k-1}\right\}+\bar{c}_{n, m, k}^{3} \operatorname{Tr}\left\{E_{; m} S^{n-k-4}\right\}+\ldots
$$

We will use the method of universal examples to show that only $\operatorname{Tr}\left\{S^{m-1}\right\}$ is relevant in computing $\left\{a_{m, m, 0}^{d+\delta}(0, g), a_{m+1, m, 1}^{d+\delta}(0, g)\right\}$ and that only $\operatorname{Tr}\left\{E_{; m} S^{m-3}\right\}$ is relevant in computing $a_{m+1, m, 0}^{d+\delta}(0, g)$. This will enable us to show:

\section{Lemma 4.2.}

(1) If $m \geq 2$, then $c_{m+1, m, 1}^{0}=\bar{c}_{m+1, m, 1}^{0}$ and $\bar{c}_{m, m, 0}^{0}=\frac{1}{(m-1) ! \operatorname{vol}\left(S^{m-1}\right)}$.

(2) $c_{4,3,0}^{3,0}=0$. If $m>3$, then $c_{m+1, m, 0}^{3,0}=\bar{c}_{m+1, m, 0}^{3}$.

Remark: The constants $\bar{c}_{n, m, k}^{0}$ and $\bar{c}_{n, m, k}^{3}$ have been determined in [5]; after a bit of work converting from $\Gamma$ functions into volumes of spheres one checks the value of $\bar{c}_{m+1, m, 1}^{0}$ given here is consistent with the value given in [5]; this provides a valuable check on our methodology.

Proof. We shall prove Lemma 4.2 by making a special case calculation. Let $m \geq 2$. To simplify the notation, let

$$
P_{m}(g):=a_{m+1, m, 1}^{d+\delta}(0, g), \quad c_{m}:=c_{m+1, m, 1}^{0}, \quad \bar{c}_{m}:=\bar{c}_{m+1, m, 1}^{0} .
$$

Let $\left(y_{1}, \ldots, y_{m-1}\right)$ be the usual coordinates on $\mathbb{R}^{m-1}$. Let $f(y)$ be a smooth even function function of $y$ and let

$$
M_{m}:=\left\{(y, r) \in \mathbb{R}^{m}: r \geq f(y)\right\} .
$$

Let $\left\{A_{1}, \ldots, A_{m-1}\right\}$ be distinct real constants. We choose $f$ so that

$$
f(0)=0, \quad\left(\partial_{i}^{y} f\right)(0)=0, \quad \text { and } \quad\left(\partial_{i}^{y} \partial_{j}^{y} f\right)(0)=A_{i} \delta_{i j} .
$$


Give $\mathbb{R}^{m}$ the usual flat metric. Then $L_{i j}(0)=-A_{i} \delta_{i j}$. We use Lemma 1.3 to compute:

$$
P_{m}(g)(0)=(m-1) ! c_{m} \mathcal{A} \quad \text { where } \mathcal{A}:=(-1)^{m-1} A_{1} \ldots A_{m-1} .
$$

Because $R=0$, we have $E=0$ and $\Omega=0$. Thus there exists a polynomial $Q_{m}$ of total weight $m-1$ in the tangential covariant derivatives of $\{\chi, L, S\}$ so that

$$
P_{m}=\sum_{p}(-1)^{p} \operatorname{Tr}_{\Lambda^{p}\left(\mathbb{R}^{m}\right)}\left\{Q_{m}(\cdot)\right\} .
$$

We must control $\tilde{\nabla}^{k} L$ for $k \geq 1$. Since the curvature of $\mathbb{R}^{m}$ vanishes, Equation (3.f) shows that $\tilde{\nabla} L$ is a totally symmetric tensor field. Since $f$ is an even function, $\tilde{\nabla}^{k} L$ vanishes at the origin if $k$ is odd. For $k$ even, the components of $\tilde{\nabla}^{k} L(0)$ are polynomials in the derivatives of the defining function $f$. Let $\mathfrak{K}$ denote the ideal in the algebra of all polynomials in the jets of $f$ which is generated by the monomials $\left\{A_{1}^{2}, \ldots, A_{m-1}^{2}\right\}$. In light of Equation (4.C), we shall work modulo $\mathfrak{K}$ since such elements can not contribute to $\mathcal{A}$.

We first study $\tilde{\nabla}^{2} L$. This is not a symmetric tensor field. Let $\tilde{R}$ be the curvature of the Levi-Civita connection of $\partial M$. Let $\left\{e_{1}, \ldots, e_{m-1}\right\}$ be an orthonormal frame for the tangent bundle of the boundary so that $e_{i}(0)=\partial_{i}^{y}$. Then:

$$
\begin{aligned}
& \tilde{R}_{b_{1} b_{2} b_{3} b_{4}}=L_{b_{1} b_{4}} L_{b_{2} b_{3}}-L_{b_{1} b_{3}} L_{b_{2} b_{4}}, \quad \text { and } \\
& L_{a_{1} a_{2}: a_{3} a_{4}}-L_{a_{1} a_{2}: a_{4} a_{3}}=\tilde{R}_{a_{3} a_{4} a_{1} a_{5}} L_{a_{5} a_{2}}+\tilde{R}_{a_{3} a_{4} a_{2} a_{5}} L_{a_{5} a_{1}} .
\end{aligned}
$$

This shows that $A_{a_{5}}^{2}$ divides $\left\{\tilde{R}_{a_{3} a_{4} a_{1} a_{5}} L_{a_{5} a_{2}}+\tilde{R}_{a_{3} a_{4} a_{2} a_{5}} L_{a_{5} a_{1}}\right\}(0)$. Consequently $\tilde{\nabla}^{2} L(0)$ is totally symmetric modulo the ideal $\mathfrak{K}$. Since the components of $\tilde{\nabla}^{2} L$ are linear in the 4 jets of $f$ and quadratic in the 2 jets of $f$, we may choose the 4 jets of $f$ to kill the symmetrization of $\left(\tilde{\nabla}^{2} L\right)(0)$ and thereby ensure $\left(\tilde{\nabla}^{2} L\right)(0) \in \mathfrak{K}$. Similarly, by choosing $\tilde{\nabla}^{k+2} f(0)$ appropriately, we may suppose that

$$
\left(\tilde{\nabla}^{k} L\right)(0) \in \mathfrak{K} \quad \text { for } \quad k>0 .
$$

We therefore supress $\tilde{\nabla}^{k} L$ henceforth in the proof of Assertions (1) and (2). By Lemma 2.2 (5), $\chi_{: a}=2 L_{a b}\left(\mathfrak{e}_{b} \mathfrak{i}_{m}+\mathfrak{e}_{m} \mathfrak{i}_{b}\right)$. Thus further covariant differentiation of $\chi$ only involves covariantly differentiating $\mathfrak{e}_{b} \mathfrak{i}_{m}+\mathfrak{e}_{m} \mathfrak{i}_{b}$. Thus inductively there exist suitably chosen endomorphisms $\mathcal{E}_{\star}$ of weight 0 so:

$$
\chi_{: a_{1} \ldots a_{k}}=L_{a_{1} b_{1}} L_{a_{2} b_{2} \ldots L_{a_{k} b_{k}}} \mathcal{E}_{b_{1} \ldots b_{k}} .
$$

If a $\chi: a_{1} \ldots$ term appears, we must contract it with another index $a_{1}$; Equation (4.d) contains no $L_{a_{1} a_{1}}$ term. Consequently this contraction involves a different variable which produces an $A_{a_{1}}^{2}$ term; such terms can be ignored in light of Equation (4.C). Similarly since

$$
S=-L_{a b} \mathfrak{e}_{b} \mathfrak{i}_{a} \text { on } \Lambda\left(\mathbb{R}^{m-1}\right) \quad \text { and } \quad S=0 \text { on } \Lambda\left(\mathbb{R}^{m-1}\right) \wedge d r,
$$

$\tilde{\nabla}^{k} S$ plays no role if $k \geq 1$. If a $L_{a_{1} b_{1}}$ term appears where $a_{1}$ is not to be contracted with $b_{1}$, then $A$ must be divisible by $A_{a_{1}}^{2}$. If the term $L_{a a}$ appears in a monomial $Q$, then we may factor $Q=L_{a a} Q_{0}$ and then apply Lemma 3.7(1) to see the supertrace of $Q_{0}$ vanishes. Thus $L$ does not appear as a variable. This shows that only the monomial $S^{m-1}$ is relevant. Consequently

$$
P_{m}(g)(0)=\bar{c}_{m} \sum_{p}(-1)^{p} \operatorname{Tr}_{\Lambda^{p}\left(\mathbb{R}^{m}\right)}\left\{S^{m-1}\right\}(0) .
$$

Since $S$ is zero on $\Lambda^{p}\left(\mathbb{R}^{m-1}\right) \wedge d r$,

$$
P_{m}(g)(0)=\bar{c}_{m} \sum_{p}(-1)^{p} \operatorname{Tr}_{\Lambda^{p}\left(\mathbb{R}^{m-1}\right)}\left\{S^{m-1}\right\}(0) .
$$


We may decompose

$$
\begin{aligned}
& \Lambda\left(\mathbb{R}^{m-1}\right)=\Lambda(\mathbb{R}) \otimes \ldots \otimes \Lambda(\mathbb{R}) \quad \text { and } \\
& S=\sum_{1 \leq i \leq m-1} \operatorname{Id} \otimes \ldots \otimes \operatorname{Id} \otimes S_{i} \otimes \operatorname{Id} \otimes \ldots \otimes \operatorname{Id} \quad \text { where } \\
& S_{i}=0 \text { on } \Lambda^{0}(\mathbb{R}) \text { and } S_{i}=-A_{i} \text { on } \Lambda^{1}(\mathbb{R}) .
\end{aligned}
$$

The supertrace of Id is zero. Furthermore, the supertrace of the tensor product is the product of the supertraces. Thus only $(m-1) ! S_{1} \otimes \ldots \otimes S_{m-1}$ survives in the supertrace of $S^{m-1}$. Since the supertrace of $S_{i}$ is $-A_{i}$, we have that:

$$
\sum_{p}(-1)^{p} \operatorname{Tr}_{\Lambda^{p}\left(\mathbb{R}^{m-1}\right)}\left\{S^{m-1}\right\}=(m-1) ! \mathcal{A} .
$$

Assertion (1) part one now follows from Equations (4.C), (4.e), and (4.f).

The invariant $a_{m, m, 0}^{d+\delta}$ is homogeneous of weight $m-1$ and is in the kernel of $r$. Thus we can use exactly the same line of argument to show:

$$
a_{m, m, 0}^{d+\delta}(0, g)(0)=(m-1) ! \mathcal{A} \bar{c}_{m, m, 0}^{0} .
$$

We use Theorem 1.1 to evaluate $a_{m, m, 0}^{d+\delta}(0, g)(0)$ and establish Assertion (1) part two.

The proof of Assertion (2) is similar. Let $m \geq 3$. To simplify the notation, set

$$
P_{m+1}(g):=a_{m+1, m, 0}^{d+\delta}(0, g), \quad c_{m+1}:=c_{m+1, m, 0}^{3,0}, \quad \text { and } \quad \bar{c}_{m+1}:=\bar{c}_{m+1, m, 0}^{3} .
$$

Let $\left(u_{1}, u_{2}, y_{1}, \ldots, y_{m-3}, r\right)$ be coordinates on $\mathbb{R}^{m}$. Let $f(y)$ satisfy the normalizations of Equation (4.b). We set $M=\left\{x \in \mathbb{R}^{m}: r \geq f(y)\right\}$ and

$$
d s_{M}^{2}:=d u_{1}^{2}+e^{-A_{0} u_{1}^{2} r} d u_{2}^{2}+d y_{1}^{2}+\ldots+d y_{m-3}^{2}+d r^{2} .
$$

Then $R(\cdot)(0)=0$ and the non-vanishing components of $L$ and $\nabla R$ at the origin are given, up to the usual $\mathbb{Z}_{2}$ symmetries, by:

$$
\begin{aligned}
& L\left(\partial_{i}^{y}, \partial_{j}^{y}\right)(0)=-A_{i} \delta_{i j}, \quad \text { and } \\
& R\left(\partial_{1}^{u}, \partial_{2}^{u}, \partial_{2}^{u}, \partial_{1}^{u} ; \partial_{r}\right)=R\left(\partial_{1}^{u}, \partial_{2}^{u}, \partial_{2}^{u}, \partial_{r} ; \partial_{1}^{u}\right)=A_{0} .
\end{aligned}
$$

Let $\mathcal{A}=(-1)^{m-3} A_{0} A_{1} \ldots A_{m-3}$. We apply Lemma 1.3 to see

$$
P_{m+1}(g)(0)=2(m-3) ! c_{m+1} \mathcal{A} .
$$

We now let $\mathfrak{K}$ be the ideal generated by the elements $\left\{A_{0}^{2}, A_{1}^{2}, \ldots, A_{m-3}^{2}\right\}$. If we set $A_{0}=0$, then the manifold is a product of the manifold considered previously with a flat factor. This shows that $\nabla^{k} R(0), \nabla^{k} E(0), \nabla^{k} \Omega(0)$ are all divisible by $A_{0}$ for $k \geq 1$ and vanish if $k=0$.

We consider terms which can give rise to $\mathcal{A}$ after taking the supertrace. Let $\mathcal{E}$ denote a generic polynomial in the tangential covariant derivatives of $L$, of $S$, and of $\chi$ when $A_{0}$ is set to zero. Since we are not interested in terms which are divisible by $A_{0}^{2}$ and since $A_{0}$ has weight 3 , we factor out a term which can be linear in $A_{0}$ to express $P_{m+1}$ symbolically as:

$$
\begin{aligned}
P_{m+1} & =\sum_{p}(-1)^{p} \operatorname{Tr}_{\Lambda^{p}(M)}\left\{\sum_{k \geq 1} \nabla^{k} R \cdot \mathcal{E}_{m-k-2}^{R}+\sum_{k \geq 1} \nabla^{k} E \cdot \mathcal{E}_{m-k-2}^{E}\right. \\
& +\sum_{k \geq 1} \nabla^{k} \Omega \cdot \mathcal{E}_{m-k-2}^{\Omega}+\sum_{k \geq 2} \tilde{\nabla}^{k} L \cdot \mathcal{E}_{m-k-1}^{L} \\
& \left.+\sum_{k \geq 2} \tilde{\nabla}^{k} S \cdot \mathcal{E}_{m-k-1}^{S}+\sum_{k \geq 3} \tilde{\nabla}^{k} \chi \cdot \mathcal{E}_{m-k}^{\chi}\right\} .
\end{aligned}
$$


We set $A_{0}=0$ in studying the 'coefficient' monomials $\mathcal{E}$. Thus the arguments given above in the proof of Assertion (1) shows only powers of $S$ are relevant so

$$
\begin{aligned}
P_{m+1} & =\sum_{p}(-1)^{p} \operatorname{Tr}_{\Lambda^{p}(M)}\left\{\sum_{k \geq 1} \nabla^{k} R \cdot S^{m-k-2}+\sum_{k \geq 1} \nabla^{k} E \cdot S^{m-k-2}\right. \\
& +\sum_{k \geq 1} \nabla^{k} \Omega \cdot S^{m-k-2}+\sum_{k \geq 2} \tilde{\nabla}^{k} L \cdot S^{m-k-1} \\
& \left.+\sum_{k \geq 2} \tilde{\nabla}^{k} S \cdot S^{m-k-1}+\sum_{k \geq 3} \tilde{\nabla}^{k} \chi \cdot S^{m-k}\right\} .
\end{aligned}
$$

By Lemma 3.7

$$
\sum_{p}(-1)^{p} \operatorname{Tr}_{\Lambda^{p}(M)}\left\{S^{k}\right\}=0 \quad \text { for } \quad k<m-1 .
$$

Thus the terms in $\nabla^{k} R$ and $\tilde{\nabla}^{k} L$ do not appear in Equation (4.h) since, being scalars, they could be moved outside Tr. As $\Omega$ is skew-adjoint and $S$ is self-adjoint, this term does not appear. Terms involving $\tilde{\nabla}^{k} S$ must be fully contracted and, modulo lower order terms which can be absorbed at an earlier stage, have the form:

$$
S_{: a_{1} a_{1} a_{2} a_{2} \ldots} S^{k}=\frac{1}{k+1}\left\{S^{k+1}\right\}_{: a_{1} a_{1} a_{2} a_{2} \ldots}+O\left(A_{0}^{2}\right) .
$$

Thus by Equation (4.i) such terms do not arise in Equation (4.h). A similar argument can be used to eliminate the terms $\chi_{: a_{1} a_{1} a_{2} a_{2} \ldots} S^{k}$ from Equation (4.h).

Extend $S$ to be covariant constant along the geodesic normal rays from the boundary. This permits us to move covariant derivatives outside the trace once again. We apply Lemma 3.7 to see

$$
\sum_{p}(-1)^{p} \operatorname{Tr}_{\Lambda^{p}(M)}\left\{E S^{k}\right\}=0 \quad \text { for } \quad k<m-3 .
$$

Thus exactly one covariant derivative of $E$ can appear and Equation (4.h) becomes

$$
P_{m+1}(g)(0)=\bar{c}_{m+1} \sum_{p}(-1)^{p} \operatorname{Tr}_{\Lambda^{p}\left(\mathbb{R}^{m}\right)}\left\{E_{; m} S^{m-3}\right\}(0) .
$$

If $m=3$, then $\sum_{p}(-1)^{p} \operatorname{Tr}_{\Lambda^{p}\left(\mathbb{R}^{3}\right)}\{E\}=0$. This implies

$$
\sum_{p}(-1)^{p} \operatorname{Tr}_{\Lambda^{p}\left(\mathbb{R}^{3}\right)}\left\{E_{; m}\right\}=0
$$

and hence $c_{m+1}=0$ as desired.

Suppose that $m \geq 4$. Since $S$ vanishes on $\Lambda\left(\mathbb{R}^{m-1}\right)^{\perp}$, we have

$$
\left.P_{m+1}(g)(0)=\bar{c}_{m+1} \sum_{p}(-1)^{p} \operatorname{Tr}_{\Lambda^{p}\left(\mathbb{R}^{m-1}\right)}\left\{E_{; m} S^{m-3}\right)\right\}
$$

We may decompose $\Lambda\left(\mathbb{R}^{m-1}\right)=\Lambda\left(\mathbb{R}^{2}\right) \otimes \Lambda\left(\mathbb{R}^{m-3}\right)$ to express $E_{; m}=\tilde{E} \otimes \mathrm{Id}$ and $S=\operatorname{Id} \otimes \tilde{S}$. This then leads to the corresponding decomposition of the supertrace

$$
\begin{aligned}
& \sum_{p}(-1)^{p} \operatorname{Tr}_{\Lambda^{p} \mathbb{R}^{m-1}}\left\{E_{; m} S^{m-3}\right\} \\
= & \sum_{a}(-1)^{a} \operatorname{Tr}_{\Lambda^{a}\left(\mathbb{R}^{2}\right)}\left\{\tilde{E}_{; m}\right\} \cdot \sum_{b}(-1)^{b} \operatorname{Tr}_{\Lambda^{b}\left(\mathbb{R}^{m-3}\right.}\left\{\tilde{S}^{m-3}\right\} .
\end{aligned}
$$

The computation performed above shows that the supertrace of $S^{m-3}$ on $\mathbb{R}^{m-3}$ is $(-1)^{m-3}(m-3) ! A_{1} \ldots . A_{m-3}$. A direct calculation of the supertrace of $E_{; m}$ on $\mathbb{R}^{2}$ yields $2 A_{0}$. The final assertion of Lemma 4.2 now follows.

We continue our study by using the various functorial properties to show:

Lemma 4.3. (1) $\bar{c}_{n, m, k}^{i}=(4 \pi)^{-(m-1) / 2} \bar{c}_{n, 1, k}^{i}$.

(2) If $n \geq 3$, then $\bar{c}_{n, m, 1}^{0}=\frac{1}{2} \bar{c}_{n, m, 0}^{0}$.

(3) If $n \geq 5$, then $\bar{c}_{n, m, 0}^{3}=\bar{c}_{n-2, m, 1}^{0}$. 
To prove Assertion (1), we use product formulas. Let $M_{1}=T^{m-1}$ be the torus and let $D_{1}$ be the scalar Laplacian. Since the structures are flat,

$$
a_{n, m-1}\left(x_{1}, D_{1}\right)=\left\{\begin{array}{rll}
(4 \pi)^{-(m-1) / 2} & \text { if } & n=0 \\
0 & \text { if } & n>0
\end{array}\right.
$$

Let $\left(M_{2}, D_{2}\right)=\left([0,1],-\partial_{r}^{2}\right)$. Let $M=M_{1} \times M_{2}$ and $D=D_{1}+D_{2}$. Let $\mathcal{B}=\nabla_{e_{m}}+S$ where $S$ is constant and where $e_{m}$ is the inward unit normal; $e_{m}=\partial_{r}$ when $r=0$ and $e_{m}=-\partial_{r}$ when $r=1$. An analogous argument to that which was used to establish Lemma 2.3 (4) can be used to establish the following identity from which Assertion (1) follows:

$$
\begin{aligned}
a_{n, m, k}(y, D, \mathcal{B}) & =\sum_{n_{1}+n_{2}=n} a_{n_{1}, m-1}\left(x_{1}, D_{1}\right) \cdot a_{n_{2}, 1, k}\left(y_{2}, D_{2}, \mathcal{B}\right) \\
& =(4 \pi)^{-(m-1) / 2} a_{n, 1, k}\left(y_{2}, D_{2}, \mathcal{B}\right) .
\end{aligned}
$$

In view of Assertion (1), it suffices to take $m=1$ in the proof of the remaining assertions. We use results from [6]. Let $M:=[0,1]$ and let $D_{0}:=-\partial_{r}^{2}$. We choose $f$ so that $f$ vanishes identically near $r=1$ so only the component $r=0$ where $\partial_{r}$ is the inward unit normal is relevant. To prove Assertion (2), we consider a conformal variation $D_{\varepsilon}:=e^{-2 \varepsilon f} D_{0}$. Then:

$$
\left.\partial_{\varepsilon} S\right|_{\varepsilon=0}=-\frac{1}{2} f_{; m} \quad \text { and }\left.\quad \partial_{\varepsilon} a_{n}\left(1, D_{\varepsilon}\right)\right|_{\varepsilon=0}=(1-n) a_{n}\left(f, D_{0}\right) .
$$

For $n \geq 3, f_{; m} S^{n-2}$ arises from no other term. Thus we may show $\bar{c}_{n, 1,0}^{0}=\frac{1}{2} \bar{c}_{n, 1,0}^{0}$ by computing:

$$
\begin{aligned}
& \partial_{\varepsilon} a_{n}\left(1, D_{\varepsilon}\right)=\left.\partial_{\varepsilon} \int_{\partial M} \bar{c}_{n, 1,0}^{0} S^{n-1} d y\right|_{\varepsilon=0}+\ldots \\
= & -\frac{1}{2}(n-1) \bar{c}_{n, 1,0} \int_{\partial M} f_{; m} S^{n-2} d y+\ldots \\
= & (1-n) a_{n}\left(f, D_{0}\right)=(1-n) \int_{\partial M} f_{; m} S^{n-2} d y+\ldots
\end{aligned}
$$

To prove Assertion (3), we consider a scalar variation $D_{\varrho}:=D_{0}-\varrho f$. We have:

$$
\left.\partial_{\varrho} a_{n}\left(1, D_{\varrho}\right)\right|_{\varrho=0}=a_{n-2}\left(f, D_{0}\right) \text {. }
$$

If $n \geq 5$, then this is the only way a term involving $f_{; m} S^{n-4}$ can arise. We show $\bar{c}_{n, 1,0}^{3}=\bar{c}_{n-2,1,1}^{0}$ by computing:

$$
\begin{aligned}
& \left.\partial_{\varrho} a_{n}\left(1, D_{\varrho}\right)\right|_{\varrho=0}=\left.\partial_{\varepsilon} \int_{\partial M} \bar{c}_{n, 1,0}^{3} E_{; m} S^{n-4} d y\right|_{\varrho=0}+\ldots \\
= & a_{n-2}\left(f, D_{0}\right)=\int_{\partial M} \bar{c}_{n-2,1,1}^{0} f_{; m} S^{n-4} d y+\ldots
\end{aligned}
$$

Remark: Lemma 4.3 (2) fails if $n=2$ and Lemma 4.3 (3) fails if $n=4$ as there are interior terms which also contribute to the variational formulae. 


\section{Proof of Theorem 1.2}

We use Lemmas 4.14 .2 and 4.3 to determine the constants of Lemma 1.3

$$
\begin{aligned}
& c_{m+1, m}=\frac{1}{\sqrt{\pi} 8^{\bar{m}} \pi^{\bar{m}} \bar{m} !} \quad \text { for } \quad m=2 \bar{m}+1, \\
& c_{m+1, m, 1}^{k}=\frac{1}{\pi^{k} 8^{k} k !} c_{m-2 k+1, m-2 k, 1}^{0}=\frac{1}{\pi^{k} 8^{k} k !} \bar{c}_{m-2 k+1, m-2 k, 1}^{0} \\
& =\frac{1}{2 \pi^{k} 8^{k} k !} \bar{c}_{m-2 k+1, m-2 k, 0}^{0}=\frac{2 \sqrt{\pi}}{2 \pi^{k} 8^{k} k !} \bar{c}_{m-2 k+1, m-2 k+1,0}^{0} \\
& =\frac{\sqrt{\pi}}{8^{k} \pi^{k} k ! \operatorname{vol}\left(S^{m-2 k}\right)(m-2 k) !}, \\
& c_{m+1, m, 0}^{1, k}=\frac{1}{\pi^{k} 8^{k} k !} c_{m+1-2 k, m-2 k, 0}^{1,0}=\frac{1}{\sqrt{\pi} \pi^{k} 8^{k} k ! \operatorname{vol}\left(S^{m-2 k-2}\right)(m-2 k-2) !}, \\
& c_{m+1, m, 0}^{2, k}=\frac{1}{\pi^{k} 8^{k} k !} c_{m+1-2 k, m-2 k, 0}^{2,0}=0, \\
& c_{m+1, m, 0}^{3, k}=\frac{1}{\pi^{k} 8^{k} k !} c_{4,3,0}^{3,0}=0 \quad \text { for } \quad 2 k=m-3 \text {, } \\
& c_{m+1, m, 0}^{3, k}=\frac{1}{\pi^{k} 8^{k} k !} c_{m-2 k+1, m-2 k, 0}^{3,0}=\frac{1}{\pi^{k} 8^{k} k !} \bar{c}_{m-2 k+1, m-2 k, 0}^{3} \\
& =\frac{1}{\pi^{k} 8^{k} k !} \bar{c}_{m-2 k-1, m-2 k, 0}^{0}=\frac{1}{2 \sqrt{\pi} \pi^{k} 8^{k} k !} \bar{c}_{m-2 k-1, m-2 k-1,0}^{0} \\
& =\frac{1}{2 \sqrt{\pi} \pi^{k} 8^{k} k !} c_{m-2 k-1, m-2 k-1,0}^{0} \\
& =\frac{1}{2 \sqrt{\pi} \pi^{k} 8^{k} k ! \operatorname{vol}\left(S^{m-2 k-2}\right)(m-2 k-2) !} \text { for } 2 k<m-3 \text {. }
\end{aligned}
$$

\section{REFERENCES}

[1] A. A. Andrianov, N. V. Borisov and M. V. Ioffe, Factorization Method And Darboux Transformation For Multidimensional Hamiltonians, Theor. Math. Phys. 61 (1984) 1078 [Teor. Mat. Fiz. 61 (1984) 183].

[2] M. F. Atiyah, R. H. Bott, and V. K. Patodi, On the heat equation and the index theorem, Invent. Math. 19 (1973), 279-330; Errata 28 (1975), 277-280.

[3] N. Berline, N. Getzler, and M. Vergne, Heat Kernels and Dirac Operators, Grundlehren Band 298, Springer-Verlag, Berlin (1991).

[4] J.-M. Bismut, Index theory and the heat equation, in Proc. ICM Berkeley 1986, Vol. I (1987), 491-504.

[5] M. Bordag, H. Falomir, E. M. Santangelo and D. V. Vassilevich, Boundary dynamics and multiple reflection expansion for Robin boundary conditions, Phys. Rev. D 65 (2002) 064032. arXiv:hep-th/0111073.

[6] T. Branson and P. Gilkey, The Asymptotics of the Laplacian on a manifold with boundary, Comm. in PDE 15 (1990), 245-272.

[7] T. Branson, P. Gilkey, K. Kirsten, and D. Vassilevich, Heat kernel asymptotics with mixed boundary conditions, Nuclear Physics B 563 (1999), 603-626. arXiv:hep-th/9906144.

[8] S. Chern, A simple intrinsic proof of the Gauss-Bonnet formula for closed Riemannian manifolds, Ann. of Math. 45 (1944), 741-752.

[9] —, On the curvature integral in a Riemannian manifold, Ann. of Math. 46 (1945), 674684.

[10] P. Gilkey, Curvature and the eigenvalues of the Laplacian for elliptic complexes, Advances in Math, 10 (1973), 344-382.

[11] - , The boundary integrand in the formula for the signature and Euler characteristic of a Riemannian manifold with boundary, Advances in Math, 15 (1975), 334-360.

[12] - Invariance theory, the heat equation, and the Atiyah-Singer index theorem, CRC Press (1995), ISBN 0-8493-7874-4.

[13] P. Gilkey, K. Kirsten, D. Vassilevich, and A. Zelnikov, Duality symmetry of the p-form effective action and supertrace of the twisted de Rham complex, Nucl. Phys. B 648 (2003) 542-556 arXiv:hep-th/0209125. 
[14] P. Greiner, An asymptotic expansion for the heat equation, Arch. Rat. Mech. Anal. 41 (1971), 163-218.

[15] K. Kirsten, Spectral functions in mathematics and physics, Chapman \& Hall/CRC, Boca Raton, FL, (2001), ISBN 1-58488-259-X.

[16] H. P. McKean and I. M. Singer, Curvature and the eigenvalues of the Laplacian, J. Diff. Geo., 1 (1967), 43-69.

[17] R. B. Melrose, The Atiyah-Patodi-Singer Index Theorem, Res. Notes in Math. 4, A. \& K. Peters, Wellesley, Mass., 1993.

[18] V. K. Patodi, Curvature and the fundamental solution of the heat operator, J. Indian Math. Soc. 34 (1970), 269-285.

[19] R. Seeley, Analytic extension of the trace associated with elliptic boundary problems, Amer. J. Math. 91 (1969), 963-983.

[20] D. V. Vassilevich, Vector fields on a disk with mixed boundary conditions, J. Math. Phys. 36 (1995) 3174 arXiv:gr-qc/9404052.

[21] D. Vassilevich and A. Zelnikov, Discrete symmetries of functional determinants, Nucl. Phys. B 594 (2000), 501-517 arXiv:hep-th/0009084.

[22] H. Weyl, The Classical Groups, Princeton Univ. Press, Princeton, 1946.

[23] E. Witten, Supersymmetry And Morse Theory, J. Diff. Geom. 17 (1982), 661-692.

E-mail address: gilkey@darkwing.uoregon.edu, klaus.kirsten@mis.mpg.de, Klaus_Kirsten@baylor.edu, vassil@itp.uni-leipzig.de 